\title{
COMMENT
}

\section{Clinical experience on video consultation in preterm follow-up care in times of the COVID-19 pandemic}

\author{
Bilge Albayrak ${ }^{1}$, Anne-Kathrin Dathe ${ }^{1}$, Larissa Cordier ${ }^{1}$, Ursula Felderhoff-Müser ${ }^{1}$ and Britta Hüning ${ }^{1}$ \\ Pediatric Research (2021) 89:1610-1611; https://doi.org/10.1038/s41390-020-01169-9
}

Today around 15 million newborns are delivered preterm representing more than one out of ten with a trend to increase. In Germany $8.4 \%$ of children are born preterm.

Even though mortality has decreased due to high-performance medicine, the rate of morbidity remains stable regarding neurodevelopmental and respiratory outcome. ${ }^{1}$ Thus, preterm follow-up care plays an important role after discharge from the neonatal intensive care unit (NICU) to homecare. Neurological follow-up is mandatory since early detection of neurodevelopmental delays and initiation of therapy may help to improve later performances. Patients are tested at defined time points $(3,6,12$, $24,36,48$ and 60 months of corrected age) using a standardized neurodevelopmental test programme: General Movement Assessment (3 months), Hammersmith Infant Neurological Examination (3, 6 and 12 months), Bayley Scales of Infant Development Version III $(12,24$ and 36 months), Wechsler Preschool and Primary Scale of Intelligence 4th Edition (48 and 60 months), Developmental Test of Visual Perception 2 (60 months). In addition, specific tests for attention disorders, problems with inhibitory control or learning disabilities at school age, are carried out if indicated. Another important aspect of the follow-up consultation is to support parent-child interaction and to provide parents with information and practical recommendations about challenges of daily life with a preterm child (such as feeding, sleeping, behavioural problems, monitoring, drug administration). ${ }^{2}$

In times of the ongoing COVID-19 pandemic parents of preterm infants are exceptionally worried since their children suffer more often from respiratory infections, with a higher risk of rehospitalization during their first two years of life. ${ }^{1}$ A key role in protecting these children at potential risk of respiratory deterioration is social distancing since there is no vaccination or adequate therapy available for COVID-19. As a consequence, we offer families the opportunity for a consultation via video instead of an examination in person for preterm follow-up care at different ages.

A Swedish study showed that telemedicine and e-health is a helpful and supportive tool in preterm follow-up care after discharge from the NICU. ${ }^{3}$ Parents felt more secure in caring for their infant, they got timely answers to their questions and the need for scheduled and emergency visits was decreased. Furthermore Holm et al. $^{3}$ showed that telemedicine increased the rate of breastfed very preterm infants.

Comparable with home visits by neonatal nurses, as shown in the study by Holm et al., ${ }^{3}$ follow-up consultations in the hospital are time consuming, costly and challenging for families. In addition, telemedicine can be an effective tool to support families in rural areas with difficult traffic connections and less accessible social services. Recently, Dayal et al. ${ }^{4}$ proofed in a cross-sectional study that children receiving neurological consultation via telemedicine utilized significantly less high-cost hospital encounters compared to children with in-person neurological consultations.

However, its scientifically investigated benefits have not resulted in ubiquitous offering of telemedicine in follow-up care of preterm or seriously ill infants in Germany. Performances in digitalization differ widely between European States. In the Digital Economy and Society Index (DESI) from 2019 Germany ranks 12th out of 28 member states while leading countries (like Nordic countries) published some research in the field of telemedicine for neonatal patients. ${ }^{5}$

The COVID-19 pandemic forces the German health-care system to increase the use of telemedicine. Video consultations provide an opportunity to meet the needs of parents and their infants in difficult times.

To date, 30 families of preterm infants with a gestational age below 34 weeks at a corrected age of 3, 6 and 9 months and at preschool age chose video consultations for follow-up care. Four of the families had twins. Two infants required monitoring and supplemental oxygen via high flow respiratory support. Families proofed identification via identification card or health insurance card. Physicians, psychologists and/or therapists performed video consultations interdisciplinary.

After the medical history was taken, parents were subsequently instructed to position the camera and to perform the neurological examination themselves. The parents of infants were guided through different positions, such as traction attempt, held seat and held stand, axillary hanging position, floating stomach position, passive rotation and stomach position. Furthermore, the spontaneous movements of the infants were observed in their domestic environment. At preschool age, children were instructed through fine and visual motor tasks: copying forms, using a scissor to cut on a line, testing precise finger coordination by grasping, transferring and releasing paper clips into a match box. Gross motor functions were tested using regular neurological examinations, e.g., finger-nose test, walking in tandem, stance and gait.

Video consultations offer many advantages for families as well as for physicians. While guiding parents the examiner explained all observed items and findings. Parents reported to be empowered by the self-examination of their child and to learn more about the neurodevelopment. In addition, questions regarding the

\footnotetext{
${ }^{1}$ Department of Pediatrics I, Neonatology, Pediatric Intensive Care, Pediatric Neurology, University Hospital Essen, University of Duisburg-Essen, Essen, Germany
} Correspondence: Bilge Albayrak (Bilge.Albayrak@uk-essen.de)

Received: 21 August 2020 Accepted: 24 August 2020

Published online: 22 September 2020 
xamination but also everyday life issues were answered like weight gain, feeding, developmental steps and self-regulation. Physicians were able to assess fine and gross motor skills as well as cognitive function in children by visual observation. All children were calm and cooperative due to the home setting with only familiar persons. For preterm infants with demand for certain support such as additional oxygen the video consultation may be a comfortable, less stressful alternative. Furthermore, ambulance transportation is avoided and resources of the health-care system are saved. All families gave positive feedback about the possibility to consult a health-care professional without having to leave their home and therefore lowering the risk of SARS-CoV-2 virus exposure.

There are limitations of this approach as some parts of the physical examination and standardized testing beyond visual observation are impossible: testing of reflexes, muscle tone and strength, auscultation and percussion, biometric measurements as well as the use of standardized test materials and forms, the interaction between child and investigator appears inappropriate via video consultation.

However, this tool bears the potential to supplement the regular care after the COVID-19 pandemic in the today's generation of digitally native and hardware-equipped parents. The easy-accessibility of telemedicine may reach families who would otherwise be lost due to various reasons.

Based on our short-term experience, the follow-up was feasible via video consultation and the neurodevelopmental status of an infant and child was surprisingly well accessible. In times of the COVID-19 pandemic video consultations as a form of social distancing especially for risk groups is highly recommended. Future studies should evaluate parental compliance and satisfaction with video consultation for preterm follow-up care.

\section{FUNDING}

This work was funded by "Stiftung Universitätsmedizin Essen" (University medicine Essen foundation).

\section{AUTHOR CONTRIBUTIONS}

The follow-up care of the described patients was performed by B.A., B.H., L.C. and A.K.D. The first draft was written by the corresponding author B.A. The co-authors B.H., L.C., A.-K.D. and U.F.-M. revised and approved the following manuscript.

\section{ADDITIONAL INFORMATION}

Competing interests: The authors declare no competing interests.

Ethical approval: This article does not contain any studies with human participants or animals performed by any of the authors.

Publisher's note Springer Nature remains neutral with regard to jurisdictional claims in published maps and institutional affiliations.

\section{REFERENCES}

1. Priante, E. et al. Respiratory outcome after preterm birth: a long and diffucult journey. Am. J. Perinatol. 33, 1040-1042 (2016).

2. Doyle, L. W. et al. Long term follow up of high risk children: who, why and how? BMC Pediatr. 14, 279 (2014).

3. Holm, K. G. et al. Growth and breastfeeding of preterm infants receiving neonatal tele-homecare compared to hospital-based care. J. Neonatal Perinat. Med. 12, 277-284 (2019).

4. Dayal, P. et al. Hospital utilization among rural children served by pediatric neurology telemedicine clinics. JAMA Netw. Open 2, e199364 (2019).

5. European Commission, Digital scoreboard. Digital Economy and Society Index (DESI) 2019 Country Report Germany. https://ec.europa.eu/digital-single-market/en/ scoreboard/germany. Accessed 13 May 2020 (2020). 